\title{
负载阿霉素的丝蛋白纳米微球
}

\author{
杨文华 $a$ 俞淑英 ${ }^{a}$ 陈胜 ${ }^{*}, b$ 刘也卓 ${ }^{c}$ 郡正中 ${ }^{a}$ 陈新*, $a$ \\ ( $a$ 聚合物分子工程国家重点实验室 复旦大学高分子科学系 先进材料实验室 上海 200433) \\ $\left({ }^{b}\right.$ 上海交通大学医学院附属瑞金医院普外科 上海 200025) \\ ( ${ }^{c}$ 上海步克医药科技有限公司 上海 201821)
}

\begin{abstract}
摘要 丝蛋白具有良好的生物相容性, 生物可降解性以及无免疫原性. 利用丝蛋白独特的亲疏水多嵌段共聚物结构特 征和构象转变机制，通过乙醇诱导和冷冻相结合的自组装方法制备得到丝蛋白纳米微球后，再在纳米微球表面包覆阿 霓素, 成功获得了负载阿霉素的丝蛋白纳米载药微球. 该载药丝蛋白纳米微球的尺寸为 $350 \sim 400 \mathrm{~nm}$, 具有圆球形态并 且分散性能良好; 其载药率为 $4.6 \%$, 包封率大于 $90 \%$, 在磷酸缓释溶液中的释放可达 7 天以上. 此外, 研究发现其缓释 行为具有 $\mathrm{pH}$ 响应性, 在 $\mathrm{pH}=5.0$ 的磷酸缓冲溶液中的缓释量明显大于在 $\mathrm{pH}=7.4$ 的缓冲液中. 体外细胞培养结果显示, 纯丝蛋白纳米微球基本没有细胞毒性; 而负载有阿霰素的丝蛋白纳米微球能明显抑制癌细胞(Hela 细胞)的增殖, 且 24 $\mathrm{h}$ 和 $48 \mathrm{~h}$ 的培养结果表现出与单纯药物相同的药效. 因此, 该负载阿霉素的丝蛋白纳米微球在临床癌症淋巴化疗方面 具有潜在的应用价值.
\end{abstract}

关键词 蛋白质; 抗癌药物; 纳米载体; 药物缓释; 淋巴化疗

\section{Doxorubicin-Loaded Silk Fibroin Nanospheres}

\author{
Yang, Wenhua $^{a} \quad$ Yu, Shuying $^{a} \quad$ Chen, Sheng*, $^{*} \quad$ Liu, Yezhuo $^{c}$ \\ Shao, Zhengzhong ${ }^{a} \quad$ Chen, Xin ${ }^{*}, a$ \\ $\left({ }^{a}\right.$ State Key Laboratory of Molecular Engineering of Polymers, Department of Macromolecular Science, \\ Laboratory of Advanced Materials, Fudan University, Shanghai 200433) \\ $\left({ }^{b}\right.$ Department of General Surgery, Ruijin Hospital, Shanghai Jiaotong University School of Medicine, Shanghai 200025) \\ ( ${ }^{c}$ Booocle Pharmaceutical Technology Co., Ltd., Shanghai 201821)
}

\begin{abstract}
Silk protein from silkworms or spiders is a very promising biomaterial due to its renewability, nontoxicity, biocompatibility and biodegradability, so it has been widely used in biomedical and pharmaceutical fields. In this article, we report our attempt to use regenerated Bombyx mori silkworm silk fibroin (RSF) as a drug-carrier to encapsulate anti-cancer drug doxorubicin (DOX). Firstly, the pristine RSF nanospheres are prepared by using a facile and clean method developed in this laboratory previously based on the self-assembly of silk protein. In brief, after adding a small amount of ethanol into RSF solution, freezing the whole system to $-20{ }^{\circ} \mathrm{C}$ for $24 \mathrm{~h}$, and then defreezing at room temperature. These RSF nanospheres almost have no cytotoxicity because there is no additional organic solvent other than ethanol involved in the preparation process. Afterwards, the DOX-loaded RSF nanospheres with the average sizes ranging from 350 to $400 \mathrm{~nm}$ are prepared by simply mixing DOX aqueous solution and RSF nanospheres solution. The characterizations from dynamic light scattering and SEM observation show that DOX-loaded RSF nanospheres have a controllable shape and size, without apparent aggregation. The drug loading is about $4.6 \%$, the encapsulation efficiency is more than $90 \%$, and the release time of such kind of DOX-loaded RSF nanospheres is over 7 days. In addition, these DOX loaded SF-nanospheres show pH-dependent release, that is, the drug releases faster in $\mathrm{pH}=5.0$ buffer solution than that in $\mathrm{pH}=7.4$ one. The DOX-loaded in the RSF nanospheres exhibits the similar curative effect to kill or inhibit Hela cells to the free DOX after incubating these drug-loaded nanospheres with Hela cells for 24 or $48 \mathrm{~h}$. All these results, including easy preparation, good biocompatibility, suitable particle size, and considerable anti-cancer efficiency, imply that such kind of biomacromolecule based anti-cancer drug nanocarrier has a great potential for the lymphatic chemotherapy in clinical applications.

Keywords proteins; anti-cancer drugs; nanocarriers; controlled release; lymphatic chemotherapy
\end{abstract}

\section{1 引言}

在肿瘤治疗过程中，单纯使用抗癌药物进行传统的
化疗存在毒副作用大、药物代谢快速、患者痛苦大等问 题，而具有缓释作用的载药纳米粒子可在到达病灶后长

*E-mail: chenx@fudan.edu.cn (X. Chen), 13701777536@163.com (S. Chen); Tel.: 021-65642866; Fax: 021-51630300

Received August 21, 2014; published October 13, 2014.

Project supported by the Specialized Research Fund for the Doctoral Program of Higher Education, MOE of China (No. 20110071110008) and the National Natural Science Foundation of China (No. 21274028).

项目受高等学校博士学科点专项科研基金(No. 20110071110008)和国家自然科学基金(No. 21274028)资助. 
时间释放药物, 提高了药物利用率, 并能降低对正常组 织的毒副作用，因而受到了广泛关注 ${ }^{[1,2]}$.

目前作为制备缓释药物载体的材料主要是天然高 分子和一些可降解的合成高分子材料, 其中蛋白质由于 其较低的细胞毒性和较高的药物负载率而成为最具潜 力的载体材料 ${ }^{[3]}$. 丝蛋白作为一种天然的结构性蛋白质, 具有来源广泛、价格低廉、生物相容性好、免疫活性低 等优点, 被普遍认为是一种优秀的生物医用材料并已得 到广泛应用 ${ }^{[4 \sim 7]}$. 本课题组在先前的工作中发明了一种 简单绿色制备丝蛋白纳米微球的方法 ${ }^{[8]}$, 可制备出粒径 200 到 $600 \mathrm{~nm}$, 尺寸均一的丝蛋白纳米微球, 可以作为 包覆抗癌化疗药物的理想载体. 这种丝蛋白纳米微球, 其粒径大小并不适合传统的通过静脉给药的化疗体 系 ${ }^{[9]}$, 而是更适合作为新型的淋巴化疗的药物载体. 般认为, 癌症细胞的转移主要是通过两种方式, 即血管 和淋巴管. 借助淋巴系统具有吞噬粒子和多余液体等特 点, 将较大的纳米粒子通过皮下注射或者腹腔注射直接 进入淋巴系统，淋巴系统就会把吞噬的粒子带到淋巴结 处, 以达到对病灶部位定向和长期给药的目的, 从而提 高药物的利用度并降低药物对正常组织的毒副作 用 ${ }^{[10,11]}$. 已有研究显示, 在淋巴化疗中, 若采用腹腔注 射方式给药, 较大尺寸(几百纳米至几微米)的脂质体和 PLGA 微球载体相比于对应的小尺寸(小于 $200 \mathrm{~nm}$ )微球 有更好的淋巴结靶向性能 ${ }^{[12 ~ 14]}$.

本研究选择的抗癌药物是阿霉素, 它是一种广谱的 抗癌药物, 对多种肿瘤均有作用, 其作用机理是嵌入 DNA 而抑制核酸的合成 ${ }^{[15,16]}$. 与大多数抗癌药物一样, 阿霉素也具有较强的毒副作用, 特别是具有较为明显的 心脏毒性 ${ }^{[17]}$, 因此为了降低其毒副作用, 有不少对于阿 霉素负载体系的研究报道, 例如采用表面包覆了聚合物 的氧化铁通过 $\mathrm{pH}$ 敏感的化学键连接阿霉素分子 ${ }^{[18]}$, 或 采用白蛋白纳米颗粒通过化学键连接阿霉素 ${ }^{[19]}$, 或采 用壳聚糖通过亲疏水相互作用负载阿霉素 ${ }^{[20]}$, 或采用 碳纳米管通过疏水相互作用和 $\pi-\pi$ 相互作用负载阿霉 素 ${ }^{[21]}$ 等. 在本文中, 作者则采用了相对简单的表面吸附 的方法制得了负载阿霉素的丝蛋白纳米微球, 并研究了 其缓释行为和对 Hela 细胞的抑制作用.

\section{2 结果与讨论}

\section{1 负载阿霉素的丝蛋白纳米微球的制备}

在作者先前采用丝蛋白纳米微球包裹疏水性药物 紫杉醇和亲水性药物氟脲苷时, 两种药物都可以和丝蛋 白乙醇溶液均匀混合, 然后通过冷冻成球从而实现对药 物的有效包覆 ${ }^{[22,23]}$. 但是在本实验中作者发现, 当阿霉 素与丝蛋白乙醇溶液均匀混合后, 在 $-20{ }^{\circ} \mathrm{C}$ 的条件下 却不能成球, 其原因可能是阿霉素和丝蛋白之间存在较 强的相互作用, 从而阻碍了丝蛋白的自组装成球过程. 考虑到阿霉素分子带正电, 丝蛋白纳米微球表面带负
电，所以作者尝试通过静电相互作用将阿霉素包覆在丝 蛋白纳米微球的表面. 但是从图 1 可以看出，简单地将 阿霉素溶液加入丝蛋白纳米微球中效果并不好, 丝蛋白 纳米微球出现了明显的聚集. 其原因一是阿霉素的加 入，中和了丝蛋白表面的负电荷，减弱了其在溶液中稳 定存在所依赖的静电斥力; 二来由于阿霉素与丝蛋白的 作用较强, 它能起到类似 “粘结剂” 的作用而将丝蛋白 纳米微球连接在一起, 从而形成比较明显的聚集.
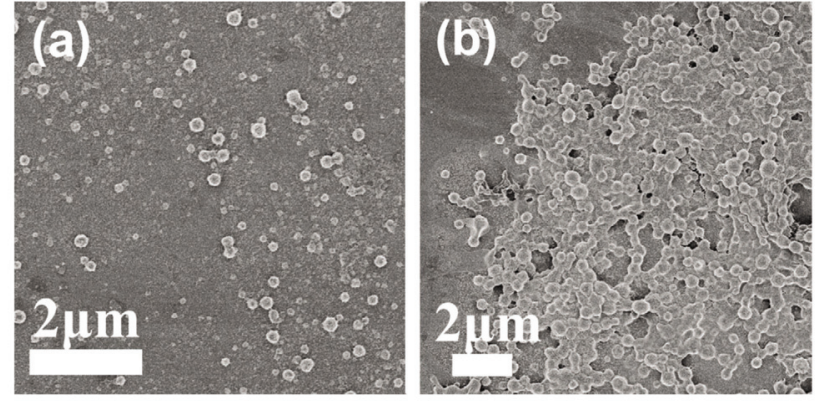

图 1 加入阿霉素前后纯丝蛋白纳米微球的扫描电镜图. (a) 加入前, (b) 加入后

Figure 1 SEM images of pure silk fibroin nanospheres before (a) and after (b) loading doxorubicin

聚乙二醇类聚合物(如聚乙二醇本身和甲氧基聚乙 二醇)常被用于稳定水溶液中的纳米微球 ${ }^{[24,25]}$, 并可使 纳米微球 “隐形”，从而避免其在达到肿瘤位点前就被 清除出去; 亦有研究表明使用聚乙二醇类聚合物能够延 长药物在体内的半衰期, 从而减少药物使用次数 ${ }^{[26,27]}$. 在本研究中, 作者使用文献中经常使用的甲氧基聚乙二 醇胺 $\left(\mathrm{mPEG}-\mathrm{NH}_{2}\right)^{[28,29]}$ 来改善丝蛋白纳米微球负载阿霉 素后的分散性(所带氨基可以和蛋白质多肽链上的基团 发生反应). 研究结果表明，加入甲氧基聚乙二醇胺后， 负载阿霉素的丝蛋白纳米微球在水溶液中的分散性得 到了明显的改善(图 2). 同时，从动态光散射测得的加入 甲氧基聚乙二醇胺所制得的丝蛋白纳米微球 (SF-PEG- $x x \%$ ，表示微球中含有 $\mathrm{xx} \%$ 的甲氧基聚乙二醇 胺)的流体力学直径和多分散指数 PDI 来看(表 1), 首先 甲氧基聚乙二醇胺的含量对丝蛋白纳米微球的尺寸影 响不大，当甲氧基聚乙二醇胺含量为 5\%到 20\%时，尺 寸几乎不变; 只有当含量增加到 $30 \%$ 时才略有增大. 其 次, 这些丝蛋白纳米微球的多分散性指数都较小. 当不 同甲氧基聚乙二醇胺含量的丝蛋白纳米微球负载阿霉 素以后，其尺寸略有增大，符合预期; 同时多分散指数 均未超过 0.2 , 进一步证实了扫描电镜的结果, 即负载 阿霉素的丝蛋白纳米微球在水溶液中的分散性良好.

由于甲氧基聚乙二醇胺含量为 $5 \%$ 时, 无论是否负 载阿霉素，丝蛋白纳米微球的分散性都非常好，而 $5 \%$ 的添加量并不会影响丝蛋白微球蛋白质的本性，所以接 下去本文都采用这个含量进行相关研究. 此外，作者在 负载阿霉素时，尝试过采用质量分数为丝蛋白 $5 \%, 6 \%$, 

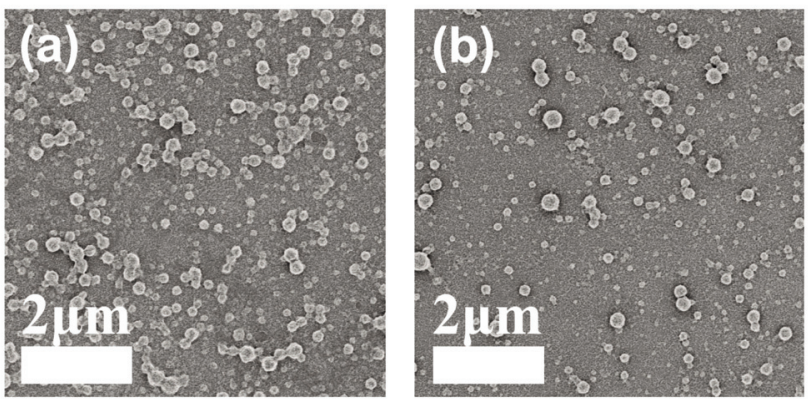

图 2 加入阿霉素前后含有甲氧基聚乙二醇胺(含量为 10\%)的丝蛋白 纳米微球的扫描电镜图. (a) 加入前, (b) 加入后

Figure 2 SEM images of $\mathrm{mPEG}-\mathrm{NH}_{2}$-contained silk fibroin nanospheres (mPEG-NH $\mathrm{NH}_{2}$ content is $10 \%$ ) before (a) and after (b) loading doxorubicin

表 1 不同甲氧基聚乙二醇胺含量的丝蛋白纳米微球负载阿露素前后 的流体力学直径、多分散性指数和 zeta 电位

Table 1 Hydrodynamic diameter, PDI and zeta-potential of DOX-loaded silk fibroin nanospheres prepared with different $\mathrm{mPEG}-\mathrm{NH}_{2}$ content

\begin{tabular}{lccc}
\hline \multicolumn{1}{c}{ Sample } & Hydrodynamic diameter/nm & PDI & Zeta-potential $/ \mathrm{mV}$ \\
\hline SF-PEG-5\% & 309 & 0.108 & -32.6 \\
SF-PEG-5\%-DOX & 356 & 0.126 & -25.8 \\
SF-PEG-10\% & 300 & 0.099 & -28.6 \\
SF-PEG-10\%-DOX & 359 & 0.185 & -24.6 \\
SF-PEG-20\% & 301 & 0.050 & -31.9 \\
SF-PEG-20\%-DOX & 341 & 0.125 & -25.6 \\
SF-PEG-30\% & 322 & 0.065 & -29.7 \\
SF-PEG-30\%-DOX & 398 & 0.168 & -24.5 \\
\hline
\end{tabular}

$7 \%$ 的阿霉素投药量, 以考察丝蛋白微球对阿霉素的最 大负载量. 结果发现, 当阿霉素的投药量为 $5 \%$ 时, 载药 微球的多分散性指数为 0.126 ; 当投药量增加到 $6 \%$ 后, 多分散性指数增大到 0.269 , 说明此时纳米微球体已出 现了一定程度的聚集; 而当投药量继续增加到 $7 \%$ 后, 体系则出现了肉眼可见的沉淀. 所以, 在本文中阿霉素 的投药量设定为 $5 \%$, 相应结果都是在这个投药量下得 到的. 此外作者通过透析法, 将未吸附在丝蛋白纳米微 球表面的阿霉素除去, 得到在投药量为 $5 \%$ 的情况下, 丝蛋白纳米微球对阿霉素的载药率为 $4.6 \%$, 负载率达 到 $91.5 \%$. 同时, 作者发现这种载药丝蛋白微球可以在 去离子水中保持稳定至少 1 个月.

从以上结果可以看出, 阿霉素与丝蛋白之间存在较 强的相互作用力. 首先阿霉素分子在水溶液中带正电, 丝蛋白纳米微球表面带有负电, 因此阿霉素可通过静电 作用吸附在丝蛋白纳米微球的表面, 从表 1 所示的丝蛋 白纳米微球负载阿霉素前后 zeta 电位的变化(zeta 电位 负值减小)证实了这一假设. 此外, 文献报道阿霉素分 子和壳聚糖分子中的氨基和羧基有一定的亲水相互作 用 ${ }^{[20]}$, 而丝蛋白分子上也存在有大量的氨基和羒基, 因 此阿霉素也可以通过亲水相互作用包覆在丝蛋白纳米
微球的表面.

\section{2 负载阿霉素的丝蛋白纳米微球的体外缓释实验}

图 3 为负载阿霉素的丝蛋白纳米微球分别在 $\mathrm{pH}=$ 5.0 和 $\mathrm{pH}=7.4$ 的磷酸缓冲溶液中的缓释曲线. 作者选 用 $\mathrm{pH} 5.0$ 缓释条件是为了模拟细胞内溶酶体和核内体 的酸性环境 ${ }^{[30]}$, 因为文献中一般认为, 纳米粒子进入细 胞是通过溶酶体和核内体, 而溶酶体和核内体的 $\mathrm{pH}$ 值 均小于 7.0, 一般认为是在 4 6.5. 所以, 如果纳米粒子 在进入癌细胞之前的缓释量小于酸性条件下的缓释量, 在治疗癌症的过程中就可以减小其对正常组织的毒副 作用. 从图 3 可以看出, 在 $\mathrm{pH}=7.4$ 和 $\mathrm{pH}=5.0$ 的两 种磷酸盐缓冲溶液中, 负载阿霉素的丝蛋白纳米微球体 系表现出了不同缓释行为. 在 $\mathrm{pH}=5.0$ 的条件下, 载药 微球在 $24 \mathrm{~h}$ 已释放出药物总量的 $70 \%$, 且总释放时间 为 7 天左右; 但是在 $\mathrm{pH}=7.4$ 的条件下, 载药微球在 24 $\mathrm{h}$ 的药物释放量只有药物总量的 $30 \%, 10$ 天后的释放量 也只达到药物总量的 $60 \%$. 负载阿霉素的丝蛋白纳米微 球之所以表现出具有 $\mathrm{pH}$ 响应的药物缓释行为, 作者认 为有两个主要因素. 首先, 阿霉素在酸性条件下的溶解 度要大于中性条件; 其次由于丝蛋白纳米微球在中性条 件下表面带负电荷, 在酸性条件下其表面的负电荷会被
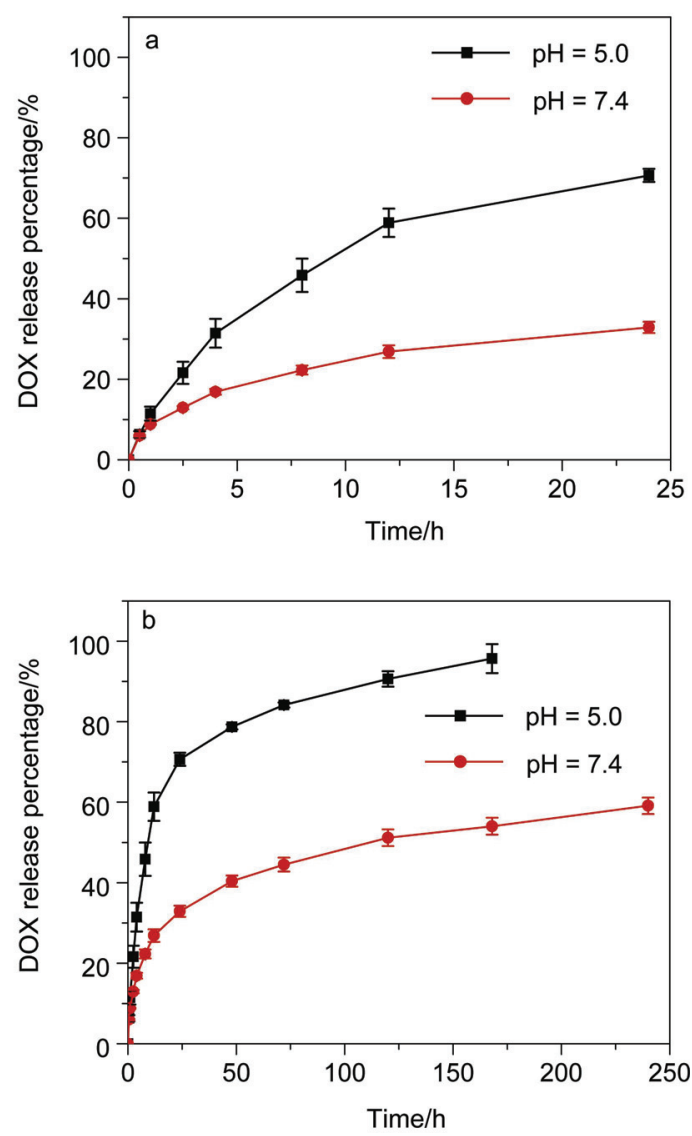

图 3 负载阿需素的丝蛋白纳米微球在 $\mathrm{pH}=5.0$ 和 $\mathrm{pH}=7.4$ 磷酸缓冲 溶液中的释放曲线. (a) $24 \mathrm{~h}$ 的释放曲线, (b) $240 \mathrm{~h}$ 的释放曲线

Figure 3 Release curves of DOX-loaded silk fibroin nanospheres at $\mathrm{pH}=5.0$ and $\mathrm{pH}=7.0$ phosphate buffer solution. (a) $24 \mathrm{~h}$, (b) $240 \mathrm{~h}$ 
中和掉一部分, 因此其对阿霉素的静电作用将会相应减 弱.

\section{3 负载阿霉素的丝蛋白纳米微球对 Hela 细胞增殖的 抑制作用}

首先作者考察了纯丝蛋白纳米微球的细胞毒性，从 图 4 可以看出, 即使丝蛋白纳米微球的浓度达到 100 $\mu \mathrm{g} / \mathrm{mL}$, 其对 Hela 细胞的细胞毒性也很小, 表明了丝蛋 白纳米微球本身具有良好的生物相容性.

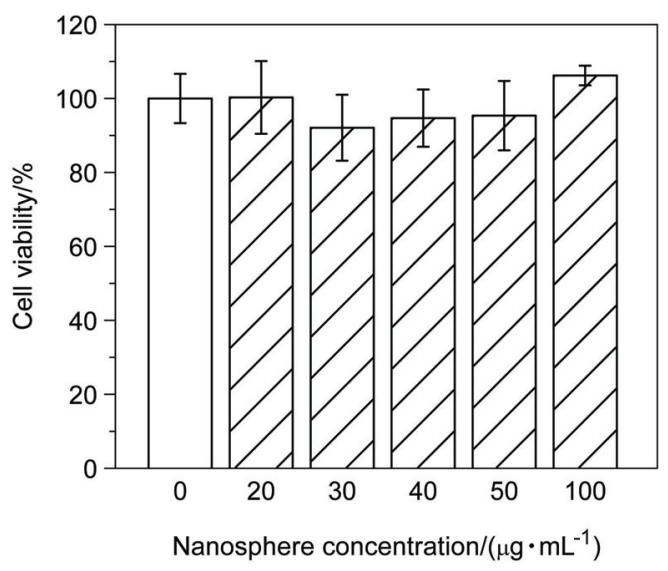

图 4 纯丝蛋白纳米微球与 Hela 细胞作用 $24 \mathrm{~h}$ 后细胞的存活率

Figure 4 Cytotoxicity of pristine silk fibroin nanospheres with Hela cells after $24 \mathrm{~h}$ incubation

图 5 为不同浓度的纯阿霉素药物和负载阿霉素的丝 蛋白纳米微球溶液分别与 Hela 细胞培养 $24 \mathrm{~h}$ 和 $48 \mathrm{~h}$ 后 Hela 细胞的存活率. 从图中可以看出, 无论是纯阿霉素 药物还是负载阿霉素的丝蛋白纳米微球, 它们对 Hela 细胞的抑制作用均随浓度的提高而增强. $24 \mathrm{~h}$ 对 Hela 细胞的半数抑制浓度(IC50)为 $0.8 \mu \mathrm{g} / \mathrm{mL}, 48 \mathrm{~h}$ 则下降为 $0.35 \mu \mathrm{g} / \mathrm{mL}$. 此外, 从这个体外实验来看, 纯阿檑素药 物和负载阿霉素的丝蛋白纳米微球的效果差不多. 但 是, 从图 3 的缓释曲线可以发现, 到 $48 \mathrm{~h}$ 其实负载在丝 蛋白纳米微球中的药物并没有释放完, 所以单纯从药物 浓度来讲, 丝蛋白纳米微球体系中阿霉素的浓度要低于 纯药物体系. 更值得注意的是我们的设想是将此载药微 球应用于淋巴化疗之中, 与本实验中很大的区别在于单 纯的药物进入体内后, 会随着血液循环或淋巴循环迅速 扩散至全身, 带来很大的毒副作用. 而如果结合淋巴化 疗进行腹腔给药注射载药微球, 由于微球对淋巴结有靶 向作用, 药物就能在病变部位有一定的富集, 从而提高 了药物的生物利用度，降低了毒副作用。

\section{3 结论}

利用丝蛋白的自组装特性, 通过乙醇诱导和冷冻相 结合的方法制备了负载阿霉素的丝蛋白纳米微球. 研究 结果表明, 加入 $5 \%$ 甲氧基聚乙二醇胺后, 可以得到形 态规整、单分散性良好, 尺寸为 $350 \sim 400 \mathrm{~nm}$ 的载药微
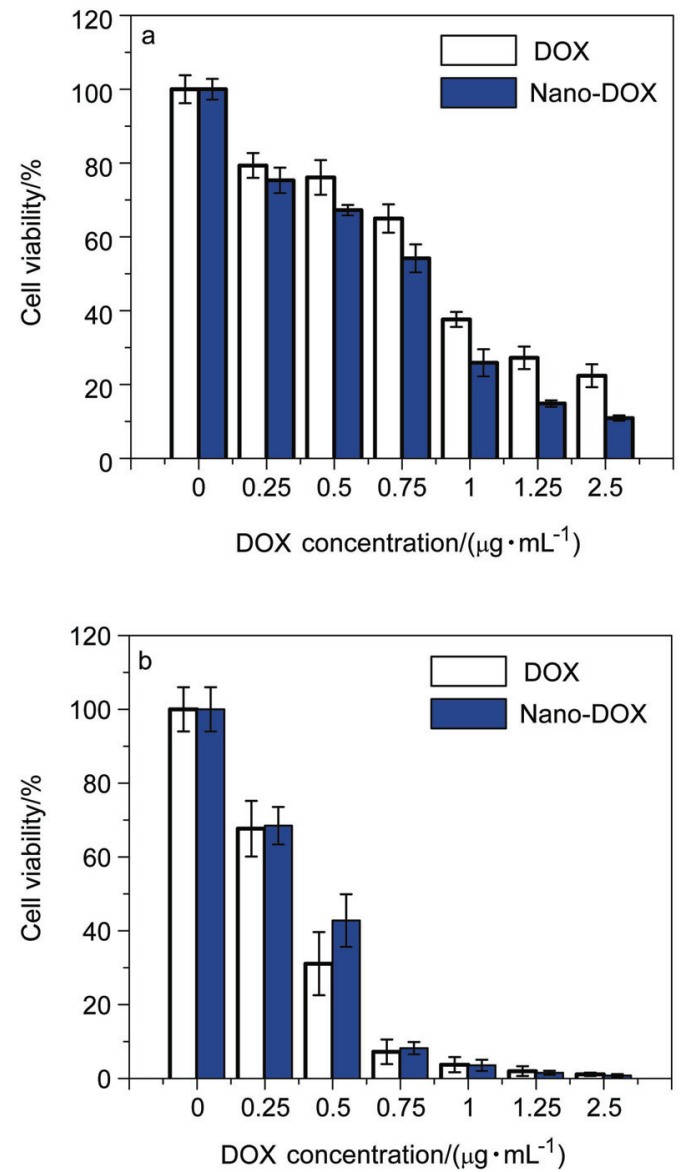

图 5 纯阿霉素药物和负载阿需素的丝蛋白纳米微球与 Hela 细胞作 用 $24 \mathrm{~h} \mathrm{(a)}$ 和 $48 \mathrm{~h}$ (b)后细胞的存活率

Figure 5 Viability of Hela cells after contacting free DOX and DOX-loaded RSF nanospheres solutions for $24 \mathrm{~h}$ (a) and $48 \mathrm{~h}$ (b)

球，其载药率为 $4.6 \%$, 包封率大于 $90 \%$. 负载阿霉素的 丝蛋白纳米微球的体外释放曲线表现出 $\mathrm{pH}$ 响应性, 即 在癌组织周围酸性条件下的释放量明显大于中性条件, 因此有利于降低药物对正常组织的毒副作用. 负载了阿 霉素的载药微球在细胞培养体系中能够实现药物的有 效释放并能明显抑制癌细胞的增殖，表明其在今后的淋 巴化疗中具有相当的应用前景.

\section{4 实验部分}

\section{1 药品与试剂}

阿霉素盐酸盐，北京药物科技有限公司; 甲氧基聚 乙二醇胺(mPEG- $\mathrm{NH}_{2}$, 分子量 2000), 阿拉丁试剂(上海) 有限公司; Hela 细胞, 中国科学院上海生命研究院细胞 资源中心.

\section{2 负载阿霉素的丝蛋白载药微球的制备}

阿霉素盐酸盐和甲氧基聚乙二醇胺分别溶解在去 离子水中，配制成 1 和 $10 \mathrm{mg} / \mathrm{mL}$ 的水溶液. 丝蛋白溶液 的制备如本课题组先前工作所述 ${ }^{[8]}$.

将甲氧基聚乙二醇胺水溶液在摚拌下缓慢滴加到 
浓度为 $40 \mathrm{mg} / \mathrm{mL}$ 丝蛋白溶液中, 然后再向其中滴加无 水乙醇, 继续轻微搅拌 $5 \mathrm{~min}$ 后置于 $-20{ }^{\circ} \mathrm{C}$ 条件下冷冻 $24 \mathrm{~h}$. 室温解冻后得到丝蛋白纳米微球乳液, 高速离心 (20000 r/min, $3 \mathrm{~min}$ )后收集沉淀, 然后再分散在去离子 水中使纳米微球的浓度为 $10 \mathrm{mg} / \mathrm{mL}$. 最后, 在丝蛋白 纳米微球乳液中边震荡边滴加阿霉素水溶液, 得到负载 阿霉素的丝蛋白纳米微球乳液.

\section{3 测试与表征}

\subsection{1 粒径和 Zeta 电势分析}

将离心分离后得到的丝蛋白纳米微球用去离子水 稀释到 $1 \mathrm{mg} / \mathrm{mL}$, 采用英国 Malvern 公司的 Zetasizer Nano 型纳米粒度-zeta 电位分析仪测定微球的粒径和多 分散性指数(PDI).

\subsection{2 扫描电镜观察}

将丝蛋白纳米微球乳液用去离子水稀释到 100 $\mu \mathrm{g} / \mathrm{mL}$ ，滴到载玻片上，自然干燥. 喷金 $15 \mathrm{~s}$ 后采用日 本 Hitachi 公司的 S-4800 高分辨场发射扫描电镜进行观 察, 测试电压为 $20 \mathrm{kV}$.

4.3.3 负载阿霉素的丝蛋白纳米微球的载药量、包封率 和体外缓释实验

取 $1 \mathrm{~mL}$ 负载阿霉素的丝蛋白纳米微球乳液于透析 袋中, 在 $5 \mathrm{~mL}$ 去离子水中透析 $1.5 \mathrm{~h}$, 每 $0.5 \mathrm{~h}$ 换新鲜 的去离子水, 以 $1.5 \mathrm{~h}$ 透析出的阿霉素总量作为未负载 到丝蛋白纳米微球上的游离阿需素量计算载药率(Drug loading)及包封率(Encapsulation efficiency). 然后将透析 袋分别放入 $5 \mathrm{~mL} \mathrm{pH}=7.4$ 和 $\mathrm{pH}=5.0$ 的磷酸盐缓冲液 中进行体外缓释实验, 每次取样后换入新鲜的磷酸盐缓 冲溶液. 采用日本 Hitachi 公司的 UV 2910 紫外/可见分 光光度计在 $480 \mathrm{~nm}$ 定波长下测定磷酸盐缓冲溶液的吸 光度，根据标准曲线计算对应的药物浓度. 丝蛋白纳米 微球的载药率和包封率的计算公式如下:

载药率 $(w t \%)=\frac{\text { 丝蛋白微球负载的药物质量 }}{\text { 丝蛋白微球的质量 }} \times 100 \%$

包封率 $(w t \%)=\frac{\text { 丝蛋白微球负载的药物质量 }}{\text { 药物的总质量 }} \times 100 \%$

\subsection{4 细胞毒性实验}

Hela 细胞以每孔 $1 \times 10^{4}$ 或 $5 \times 10^{3}$ 数量级细胞密度 种植于 96 孔板中, 分别对应于 24 和 $48 \mathrm{~h}$ 的细胞毒性实 验. 实验中设置空白组、对照组、阿霉素不同浓度组, 单 纯药物的阿霉素浓度和负载在丝蛋白纳米微球中的阿 霉素浓度分别为 $0.25,0.5,1.0,1.5,2.0,2.5 \mu \mathrm{g} / \mathrm{mL}$. 每组 实验组设置 4 个复孔. 培养基为含有 $10 \%$ 小牛血清的 DMEM 培养基. 首先将 Hela 细胞在含有 $5 \% \mathrm{CO}_{2}$, 温 度为 $37{ }^{\circ} \mathrm{C}$ 的培养箱中培育 $24 \mathrm{~h}$, 然后加入各种不同含 量阿霉素的培养液再培养 24 或 $48 \mathrm{~h}$, 之后把孔板中的
溶液换成含有 $0.5 \% \mathrm{MTT}$ 的细胞培养液，继续培养 $4 \mathrm{~h}$. 最后把培养液全部吸出，加入 $150 \mu \mathrm{L}$ 二甲基亚砜溶解 甲瓒晶体, 用酶标仪测定 $490 \mathrm{~nm}$ 处吸光度 OD 值, 根 据 OD 值计算细胞相对增殖率(RGR).

$$
R G R=\frac{\text { 试样组 } O D \text { 值一空白组 } O D \text { 值 }}{\text { 对照组 } O D \text { 值－空白组 } O D \text { 值 }} \times 100 \%
$$

\section{References}

[1] Williams, D. F. Biomaterials 2008, 29, 2941.

[2] Naahidi, S.; Jafari, M.; Edalat, F.; Raymond, K.; Khademhosseini, A.; Chen, P. J. Controlled Release 2013, 166, 182.

[3] Elzoghby, A. O.; Samy, W. M.; Elgindy, N. A. J. Controlled Release 2012, 161, 38.

[4] Wang, Y. Z.; Kim, H. J.; Vunjak-Novakovic, G.; Kaplan, D. L. Biomaterials 2006, 27, 6064.

[5] Jiang, X. R.; Guan, J.; Chen, X.; Shao, Z. Z. Acta Chim. Sinica 2010, 68, 1909. (江霞蓉, 管娟, 陈新, 邵正中，化学学报，2010, $68,1909$.

[6] Ma, X. Y.; Shi, L. J.; Zhou, J.; Zhu, J.; Zhong, J.; Wei, R. L. Chin. J. Org. Chem. 2013, 33, 1080. (马晓晔, 施丽君, 周涓, 朱君, 钟建, 魏锐利, 有机化学, 2013, 33, 1080.)

[7] Wei, Q. N.; Ma, L.; Huang, A. M.; Yang, H.; Gong, Z. P.; Qiang, P. P.; Zhang, L. Acta Chim. Sinica 2012, 70, 714. (韦俏娜, 马林, 黄 爱民, 杨华, 龚珠萍, 强盼盼, 张丽, 化学学报, 2012, 70, 714.)

[8] Cao, Z. B.; Chen, X.; Yao, J. R.; Huang, L.; Shao, Z. Z. Soft Matter $2007,3,910$.

[9] Moghimi, S. M.; Hunter, A. C.; Murray, J. C. Pharm. Rev. 2001, 53, 283.

[10] Swartz, M. A. Adv. Drug Delivery Rev. 2001, 50, 3.

[11] Oussoren, C.; Storm, G. Adv. Drug Delivery Rev. 2001, 50, 143.

[12] Liu, J.; Wong, H. L.; Moselhy, J.; Bowen, B.; Wu, X. Y.; Johnston, M. R. Lung Cancer 2006, 51, 377 .

[13] Nishioka, Y.; Yoshino, H. Adv. Drug Delivery Rev. 2001, 47, 55.

[14] Liu, J.; Meisner, D.; Kwong, E.; Wu, X. Y.; Johnston, M. R. Biomaterials 2007, 28, 3236 .

[15] Lee, S.; Baek, M.; Kim, H.-Y.; Ha, J.-H.; Jeoung, D.-I. Biotechnol. Lett. 2002, 24, 1147.

[16] He, H.; Wang, Y.; Wen, H.; Jia, X. RSC Adv. 2014, 4, 3643.

[17] Carvalho, F. S.; Burgeiro, A.; Garcia, R.; Moreno, A. J.; Carvalho, R. A.; Oliveira, P. J. Med. Res. Rev. 2014, 34, 106.

[18] Basuki, J. S.; Duong, H. T.; Macmillan, A.; Erlich, R. B.; Esser, L.; Akerfeldt, M. C.; Whan, R. M.; Kavallaris, M.; Boyer, C.; Davis, T. P. ACS Nano 2013, 7, 10175.

[19] Guo, Q. Q.; Wang, H. Y.; Zhao, Y. X.; Wang, H. X.; Zeng, F.; Hua, H. Y.; Xu, Q.; Huang, Y. Z. Polym. Chem. 2013, 4, 4584.

[20] Sanyakamdhorn, S.; Agudelo, D.; Tajmir-Riahi, H.-A. Biomacromolecules 2013, 14, 557.

[21] Liu, Z.; Sun, X. M.; Nakayama-Ratchford, N.; Dai, H. J. ACS Nano 2007, 1,50

[22] Yu, S. Y.; Yang, W. H.; Chen, S.; Chen, M. J.; Liu, Y. Z.; Shao, Z. Z.; Chen, X. RSC Adv. 2014, 4, 18171.

[23] Chen, M. J.; Shao, Z. Z.; Chen, X. J. Biomed. Mater. Res. A 2012, 100, 203.

[24] Chan, J. M.; Zhang, L. F.; Yuet, K. P.; Liao, G.; Rhee, J.-W.; Langer, R.; Farokhzad, O. C. Biomaterials 2009, 30, 1627.

[25] Cheng, J. J.; Teply, B. A.; Sherifi, I.; Sung, J.; Luther, G.; Gu, F. X.; Levy-Nissenbaum, E.; Radovic-Moreno, A. F.; Langer, R.; Farokhzad, O. C. Biomaterials 2007, 28, 869.

[26] Allen, C.; Dos Santos, N.; Gallagher, R.; Chiu, G.; Shu, Y.; Li, W.; Johnstone, S.; Janoff, A.; Mayer, L.; Webb, M. Biosci. Rep. 2002, 22,225 .

[27] Greenwald, R. B.; Choe, Y. H.; McGuire, J.; Conover, C. D. Adv. Drug Delivery Rev. 2003, 55, 217.

[28] Ma, G. L.; Zhao, S. X.; Jin, X.; Chen, M. M.; Zhang, Z. P.; Song, C. X. Chem. J. Chin. Univ. 2012, 33, 1854 (马桂蕾, 赵顺新, 金旭, 陈旼旼, 张政朴, 宋存先, 高等学校化学学报, 2012, 33, 1854.)

[29] Waku, T.; Matsusaki, M.; Kaneko, T.; Akashi, M. Macromolecules 2007, 40, 6385 .

[30] Watson, P.; Jones, A. T.; Stephens, D. J. Adv. Drug Delivery Rev. $\mathbf{2 0 0 5}, 57,43$. 